\title{
Asiatic acid inhibits left ventricular remodeling and improves cardiac function in a rat model of myocardial infarction
}

\author{
LIANYING HUO $^{1 *}$, WENBING SHI $^{*}$, LING CHONG $^{1}$, JINLONG WANG $^{2}, \mathrm{KAI} \mathrm{ZHANG}^{1}$ and YUFENG $\mathrm{LI}^{3}$ \\ ${ }^{1}$ Department of Cardiology, Shanxian Dongda Hospital, Shanxian, Shandong 274300; \\ ${ }^{2}$ Department of Cardiology, Heze Shili Hospital, Heze, Shandong 274000; \\ ${ }^{3}$ Department of Cardiology, Chinese PLA General Hospital, Beijing 100853, P.R. China
}

Received May 10, 2015; Accepted October 30, 2015

DOI: $10.3892 / \mathrm{etm} .2015 .2871$

\begin{abstract}
Left ventricular remodeling results in cardiac dysfunction and accounts for the majority of the morbidity and mortality following myocardial infarction (MI). The aim of the present study was to investigate the effect of asiatic acid (AA) on cardiac function and left ventricular remodeling in a rat model of MI and explore the underlying mechanisms. Rats were subjected to coronary artery ligation to model MI and orally treated with AA. After 4 weeks, cardiac function was assessed by echocardiography. Cardiomyocyte cross-sectional area was recorded, and the expression levels of a number of inflammatory cytokines were detected using ELISA. The degree of interstitial fibrosis was determined by evaluating the mRNA expression levels of collagen II and III. Western blot analysis was performed to detect the expression levels of total and phosphorylated p38 MAPK and ERK1/2, to investigate whether they are involved in the mechanism underlying the effect of AA on the heart. Rats subjected to MI displayed significantly impaired cardiac function compared with those subjected to a sham procedure, while this change was reversed by treatment with AA. Furthermore, AA markedly inhibited cardiac hypertrophy, reduced the mRNA expression levels of inflammatory cytokines and decreased interstitial fibrosis in the infarct border zone of MI model rats compared with those in vehicle-treated MI model rats. Furthermore, the phosphorylation of p38 MAPK and ERK1/2 was blocked by AA in the MI rats but not in the sham rats. In summary, AA treatment preserved cardiac function and inhibited left ventricular remodeling, potentially by blocking the phosphorylation of p38 MAPK and ERK1/2 in the infarct border zone of the
\end{abstract}

Correspondence to: Dr Yufeng Li, Department of Cardiology, Chinese PLA General Hospital, 28 Fuxing Road, Beijing 100853, P.R. China

E-mail: lyf301@yahoo.com.cn

*Contributed equally

Key words: asiatic acid, cardiac remodeling, interstitial fibrosis, cardiac hypertrophy, inflammation, myocardial infarction ischemic myocardium, indicating that AA may be a novel candidate for development as a therapy for MI.

\section{Introduction}

Myocardial infarction (MI) is among the leading causes of mortality worldwide (1). Patients that survive an acute MI may subsequently suffer heart failure (HF), with left ventricular remodeling as the most common underlying factor $(2,3)$. Myocardial hypertrophy, inflammation and significant fibrosis are considered to be characteristic pathological alterations of post-infarct remodeling and major determinants of the progressive deterioration of cardiac function following MI (4-7).

Generally, remodeling is a complex process modulated by a cascade of biochemical signaling processes triggered by the necrotic myocardium (8). Transforming growth factor- $\beta 1$ (TGF- $\beta 1$ ) is a key inflammatory molecule that induces cardiac fibrosis (4,9-11). Furthermore, intracellular mitogen-activated protein kinase (MAPK) signaling cascades have been demonstrated to serve a crucial function in the pathogenesis of cardiac hypertrophy and fibrosis, and inhibitors of this signaling pathway may suppress interstitial fibrosis and improve cardiac function (12). In addition, the augmentation of extracellular signal-regulated kinase 1 and 2 (ERK1/2) activity may enhance interstitial fibrosis, myocardial hypertrophy and cardiac dysfunction $(13,14)$. Therefore, agents that affect the molecular pathways elicited during post-infarct remodeling may be promising therapeutic candidates.

Asiatic acid (AA; Fig. 1) is a pentacyclic triterpenoid derived from the tropical medicinal plant Centella asiatica (Apiaceae family), and serves a variety of biological functions (15). AA has been shown to prevent ultraviolet A-mediated photoaging, facilitate wound healing and exert anti-inflammatory and anti-diabetic activities (16-19). In addition, AA has been reported to exhibit anti-fibrotic functions in the liver via abrogating the TGF- $\beta 1 /$ Smad pathway and to protect the brain from ischemic injury $(15,20)$. Previous studies have reported that AA exerts beneficial effects on the heart; for example, AA is able to inhibit pressure overload-induced cardiac hypertrophy by blocking p38 MAPK and ERK1/2 phosphorylation and reducing the excessive production of TGF- $\beta 1$ and nuclear factor (NF) $-\kappa B(21)$. In addition, AA protects cardiomyocytes against high-glucose-induced injury by attenuating oxidative 
stress (22). However, whether AA exerts a protective effect on the heart following myocardial ischemia remains unknown.

Therefore, the present study aimed to investigate the capacity of AA to inhibit p38 MAPK and ERK1/2 phosphorylation and TGF- $\beta 1$ overexpression, thus reducing cardiac inflammation and fibrosis and improving cardiac function following MI.

\section{Materials and methods}

Animals and experimental protocol. All procedures were conducted in accordance with the Guide for the Care and Use of Laboratory Animals published by the US National Institutes of Health (8th edition, 2011) and approved by the Institutional Animal Care and Use Committee of the Academy of Military Medical Science (SYXK2012-005; Beijing, China). A total of 120 adult male Sprague-Dawley rats (220-240 g) were purchased from the Experimental Animal Center, Academy of Military Medical Science. The rats were maintained in an air-conditioned room at $25 \pm 1^{\circ} \mathrm{C}$ with a 12 -h light/dark cycle, and free access to food and water. After 1 week of acclimatization, rats were allocated at random into 8 groups $(n=15$ per group): i) $\mathrm{S}+\mathrm{V}$, sham rats treated with vehicle; ii) $\mathrm{S}+\mathrm{AA}_{5}$, sham rats treated with $5 \mathrm{mg} / \mathrm{kg} /$ day $\mathrm{AA}$; iii) $\mathrm{S}+\mathrm{AA}_{25}$, sham rats treated with $25 \mathrm{mg} / \mathrm{kg} /$ day $\mathrm{AA}$; iv) $\mathrm{S}+\mathrm{AA}_{50}$, sham rats treated with $50 \mathrm{mg} / \mathrm{kg} /$ day $\mathrm{AA}$; v) $\mathrm{MI}+\mathrm{V}$, MI rats treated with vehicle; vi) $\mathrm{MI}+\mathrm{AA}_{5}$, $\mathrm{MI}$ rats treated with $5 \mathrm{mg} / \mathrm{kg} /$ day $\mathrm{AA}$; vii) $\mathrm{MI}+\mathrm{AA}_{25}$, MI rats treated with $25 \mathrm{mg} / \mathrm{kg} / \mathrm{day} \mathrm{AA}$; and viii) $\mathrm{MI}+\mathrm{AA}_{50}$, MI rats treated with $50 \mathrm{mg} / \mathrm{kg} /$ day $\mathrm{AA}$. MI was induced by permanent left anterior descending (LAD) coronary artery ligation. Sham groups underwent an identical surgical procedure without coronary ligation.

Rat model of MI and AA treatment. Male Sprague-Dawley rats were subjected to left anterior descending (LAD) coronary artery ligation to induce MI, as described previously (23). Briefly, the rats were anaesthetized with $1 \%$ sodium phenobarbital (40-60 mg/kg) intraperitoneally and intubated orally with a polyethylene tube for artificial respiration (UGO Basile S.R.L., Comerio, Italy). A thoracotomy was performed at the fourth intercostal space, and the LAD branch was ligated at $\sim 2 \mathrm{~mm}$ from its origin. Sham-operated animals underwent an identical procedure, with the exception that the coronary ligature was not ligated. Drug administration was initiated in rats that survived for $6 \mathrm{~h}$ after surgery. AA was dissolved in 5\% dimethyl sulfoxide (DMSO) to form a solution of $10 \mathrm{mg} / \mathrm{ml}$, then diluted to a volume of $2 \mathrm{ml}$ with a specific concentration determined according to the weight of each rat. The solution was administered orally to the experimental groups using an intragastric tube daily for a period of 4 weeks. The $\mathrm{S}+\mathrm{V}$ and $\mathrm{MI}+\mathrm{V}$ groups received an identical volume $(2 \mathrm{ml})$ of vehicle $(5 \%$ DMSO) once per day for 4 weeks. To the best of our knowledge, there are no prior studies associating AA with toxicity in animal models. However, previous studies have reported that dosages of 5, 25 or $50 \mathrm{mg} / \mathrm{kg} /$ day are well-tolerated by rats and have no effect on food consumption, activity, weight, mean arterial pressure and heart rate $(24,25)$. After 4 weeks of drug administration, the rats were anesthetized, and lungs were excised and immediately weighed. In addition, the hearts

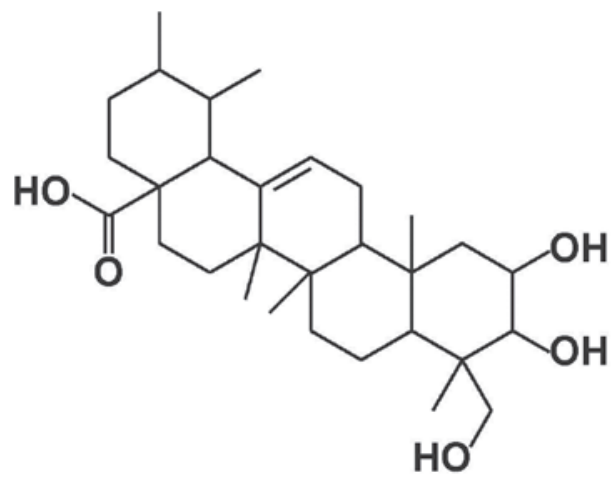

Figure 1. Chemical structure of asiatic acid.

were excised, washed in ice-cold phosphate-buffered saline (PBS) and weighed (heart weight, HW).

Echocardiography for determination of cardiac function. At the end of the 4-week drug administration period, alterations in left ventricular function were evaluated by transthoracic echocardiography using a Vivid 7 ultrasound machine (GE Healthcare Bio-Sciences, Pittsburgh, PA, USA) equipped with a $10-\mathrm{MHz}$ phased-array transducer. Left ventricular systolic diameter and left ventricular diastolic diameter were measured simultaneously, and left ventricular ejection fraction (LVEF) and fractional shortening (FS) were calculated using M-mode recording by computer algorithms (TruScan 2.07; Harvard Apparatus, Holliston, MA, USA). All measurements were calculated based on the results of three consecutive cardiac cycles and all measurements were performed in a blinded manner (26).

Determination of cardiomyocyte cross-sectional area. Following 4 weeks of drug administration, the rats were anesthetized with an intraperitoneal injection of $50 \mathrm{mg} / \mathrm{kg}$ sodium pentobarbital. Adequate anaesthesia was ensured by monitoring the absence of a withdrawal response to a paw pinch. The hearts were rapidly excised and washed in ice-cold PBS. Then the atria were removed and the left ventricle heart samples were fixed in $4 \%$ paraformaldehyde and embedded in paraffin. The heart samples were cut into 5- $\mu \mathrm{m}$ sections and then stained with hematoxylin and eosin (H\&E; AR1180-100; Wuhan Boster Biological Technology, Ltd., Wuhan, China). Conventional light microscopy (ML32; Micro-shot Technology Co., Ltd., Guangzhou, China) at x400 magnification was used to determine cardiomyocyte cross-sectional area, as previously described (27).

Determination of tissue cytokines by enzyme-linked immunosorbent assay (ELISA). The protein levels of TGF- $\beta 1$, $\mathrm{NF}-\kappa \mathrm{B}$, tumor necrosis factor (TNF) $-\alpha$ and interleukin (IL)-1 $\beta$ were determined using ELISA kits (R\&D Systems, Inc., Minneapolis, MN, USA), according to the manufacturer's instructions. In brief, myocardial tissue samples were homogenized in ice-cold PBS containing protease inhibitor cocktail (B14001; Biotool LLC, Houston, TX, USA), and total proteins were extracted using NE-PER Cytoplasmic Extraction Reagents (Pierce Biotechnology, Inc., Rockford, IL, USA). Total protein concentration (pg/mg total tissue protein) 
A

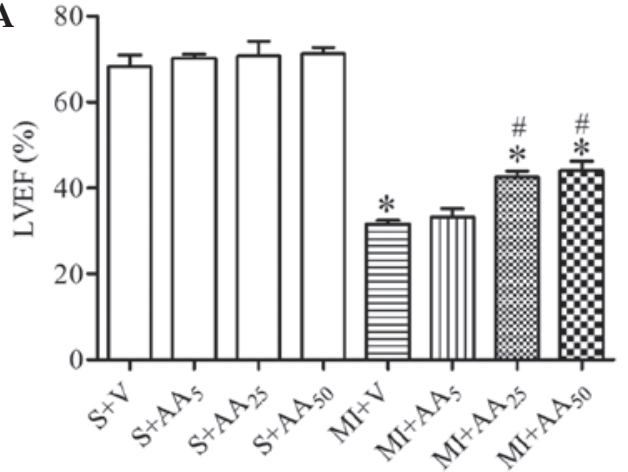

B

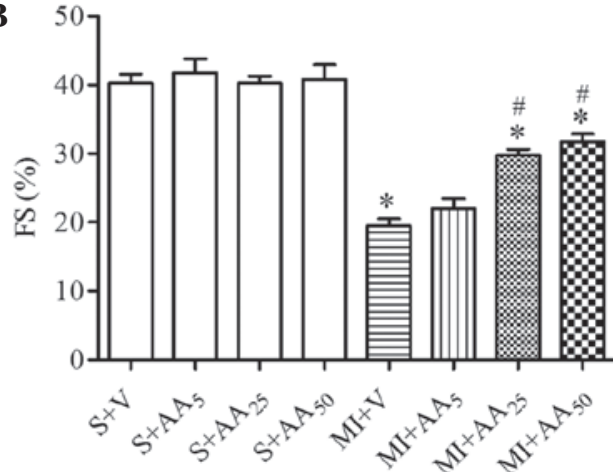

Figure 2. AA treatment improved cardiac function following MI in a dose-dependent manner. Echocardiographic analysis of cardiac (A) LVEF and (B) FS in the eight groups ( $\mathrm{n}=15$ per group) of rats at 4 weeks after MI modeling. Values are presented as the mean \pm standard deviation. ${ }^{*} \mathrm{P}<0.05$ vs. $\mathrm{S}+\mathrm{V},{ }^{\#} \mathrm{P}<0.05$ vs. MI + V. LVEF, left ventricular ejection fraction; FS, fractional shortening; S, sham; V, vehicle; AA, asiatic acid; MI, myocardial infarction.

was determined using a Bicinchoninic Acid Protein Assay kit (Beyotime Institute of Biotechnology, Shanghai, China), according to the manufacturer's instructions.

Determination of infarct size and cardiac fibrosis. To determine the infarct size of the heart and interstitial fibrosis in the infarct border zone, the collagenous fibrotic area of the heart was stained by applying Masson's Trichrome stain to $5-\mu \mathrm{m}$ paraffin-embedded sections (28). Infarct size was assessed by examining images obtained at x10 magnification using a digital camera (EOS 70D; Canon Co., Ltd., Beijing, China) and calculated as the ratio of the average scar circumference versus the average inner left ventricular circumference. The extent of cardiac fibrosis in the peri-infarct region was assessed by calculating the collagen area fraction. Quantitative evaluations were performed using ImagePro Plus 6.0 software (Media Cybernetics, Inc., Rockville, MD, USA).

Reverse transcription-quantitative polymerase chain reaction $(R T-q P C R)$. Total RNA was extracted from the cardiac myocytes and cardiac tissues using TRIzol reagent (CW0580A; CWBio Co., Ltd., Beijing, China). The cDNA was prepared using a Reverse Transcription System kit [Promega (Beijing) Biotech Co., Ltd., Beijing, China], according to the manufacturer's instructions. Following reverse transcription, the cDNA obtained was subjected to qPCR analysis to determine the mRNA expression levels of collagen I and III. qPCR was performed using predesigned Taqman Gene Expression Assays and AmpliTaq Gold DNA polymerase kits, following the manufacturer's instructions (Applied Biosystems, Foster City, CA, USA). The PCR cycling conditions were as follows: $30 \mathrm{sec}$ at $94^{\circ} \mathrm{C}, 30 \mathrm{sec}$ at $58^{\circ} \mathrm{C}$, and $30 \mathrm{sec}$ at $72^{\circ} \mathrm{C}$ (30 cycles). The mRNA levels were quantified using SYBR Green (EP1601; BioTeke Corporation, Beijing, China) and an ABI 7500 Real-Time PCR system (Applied Biosystems), and GAPDH was used as an internal control. Primers and probes were designed using Primer Express software, version 2.0 (Applied Biosystems). Primers used were as follows: Collagen I, 5'-CAGATTGGGATGGAGGGAGTT TA-3' (forward) and 5'-CTACAGCACGCTTGTGGATGG CT-3' (reverse); collagen III, 5'-ATAGCTGAACTGAAAGCC ACCAT-3' (forward) and 5'-CCTGAACTCAAGAGCGGA ATA-3' (reverse); GAPDH, 5'-GTCACCTTCACCGTTCCA
GTTTT-3' (forward) and 5'-CTTAGTTGCGTTACACCC TTTCTT-3' (reverse).

Western blot assay. Protein was isolated from homogenized heart tissue following standard protocols (29). Total proteins were loaded onto a 12\% SDS-PAGE gel (P0012A; Beyotime Institute of Biotechnology) and transferred electrophoretically to nitrocellulose membranes (EMD Millipore, Billerica, MA, USA). Following blocking with 5\% skimmed milk, the membranes were incubated with the primary antibodies (listed below) of the recommended dilution at $4^{\circ} \mathrm{C}$ overnight. The membranes were washed in Tris-buffered saline and Tween 20 (three times for $10 \mathrm{~min}$ ) and further incubated with horseradish peroxidase-linked secondary antibody at $37^{\circ} \mathrm{C}$ for $60 \mathrm{~min}$. The blots were developed using an enhanced chemiluminescence reagent kit (EMD Millipore) and visualized using a Bio-Imaging System (BioSpectrum 510; UVP, LLC, Upland, CA, USA). VisionWorks LS Acquisition and Analysis Software were used to analyze blot densities (UVP, LLC).

Primary antibodies used in this study were as follows: Polyclonal rabbit anti-human ERK1/2 antibody (1:2,000; sc-292838; Santa Cruz Biotechnology, Inc., Santa Cruz, CA, USA), monoclonal mouse anti-human p-ERK1/2 antibody (1:500; sc-81492; Santa Cruz Biotechnology, Inc.), monoclonal rabbit anti-human p38 MAPK antibody (1:1,000; \#8690; Cell Signaling Technology, Inc., Danvers, MA, USA), monoclonal rabbit anti-human phospho-p38 MAPK antibody $(1: 1,000$; \#4511; Cell Signaling Technology, Inc.) and monoclonal mouse anti-human $\beta$-actin antibody (1:5,000; KC-5A08; KangChen Bio-tech Co., Ltd., Shanghai, China). Secondary antibodies were polyclonal goat anti-rabbit (1:1,000; HAF008; R\&D systems, Inc.) and polyclonal goat anti-mouse (1:1,000; HAF007; R\&D systems, Inc.).

Statistical analysis. Continuous variables that approximated the normal distribution were expressed as mean \pm standard deviation. Comparisons between multiple groups were performed using analysis of variance followed by Bonferroni correction for post hoc t-test. Two-sided tests were used throughout, and $\mathrm{P}<0.05$ was considered to indicate a statistically significant difference. SPSS software, version 14.0 (SPSS, Inc., Chicago, IL, USA) was used for data analysis. 
A

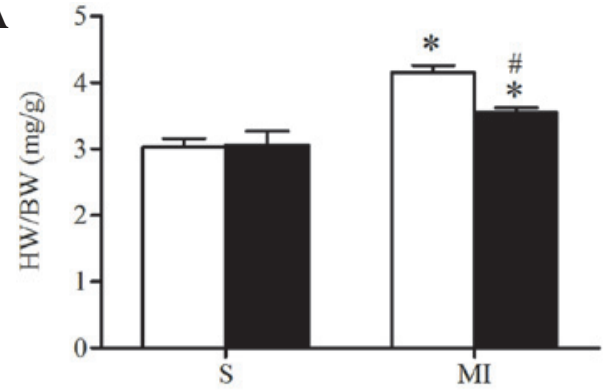

C

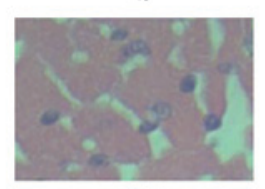

MI

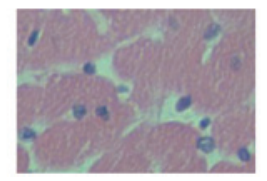

$\mathrm{S}+\mathrm{AA}_{25}$

$\mathrm{MI}+\mathrm{AA}_{25}$

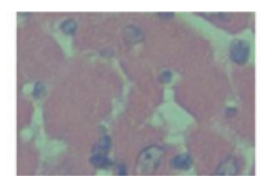

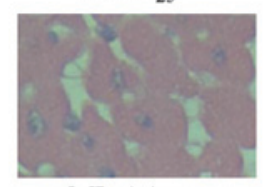

B
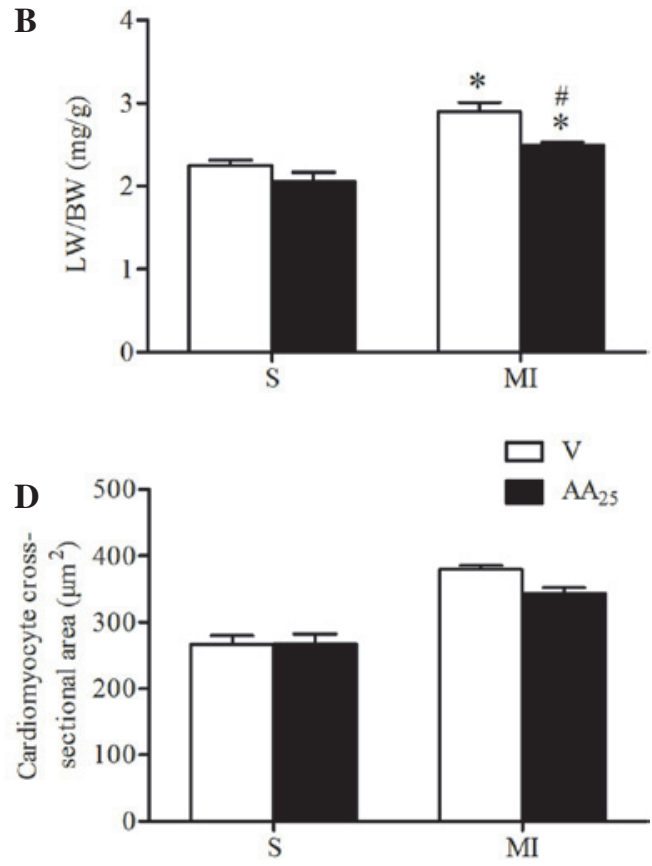

Figure 3. AA prevented cardiac hypertrophy following MI. (A) HW/BW ratio and (B) LW/BW ratio in rats from the four groups (n=8-15 per group) at 4 weeks after MI modeling. (C) Representative images of hematoxylin and eosin-stained heart sections in rats at 4 weeks after MI (magnification, $\mathrm{x} 400$ ). (D) Quantitative analysis of cardiomyocyte cross-sectional area. Values are presented as the mean \pm standard deviation. ${ }^{*} \mathrm{P}<0.05 \mathrm{MI}$ vs. $\mathrm{S}$ group; ${ }^{\#} \mathrm{P}<0.05$ $\mathrm{AA}_{25}$ vs. V group. S, sham; HW, heart weight; BW, body weight; LW, lung wet weight; MI, myocardial infarction; V, vehicle; AA, asiatic acid.
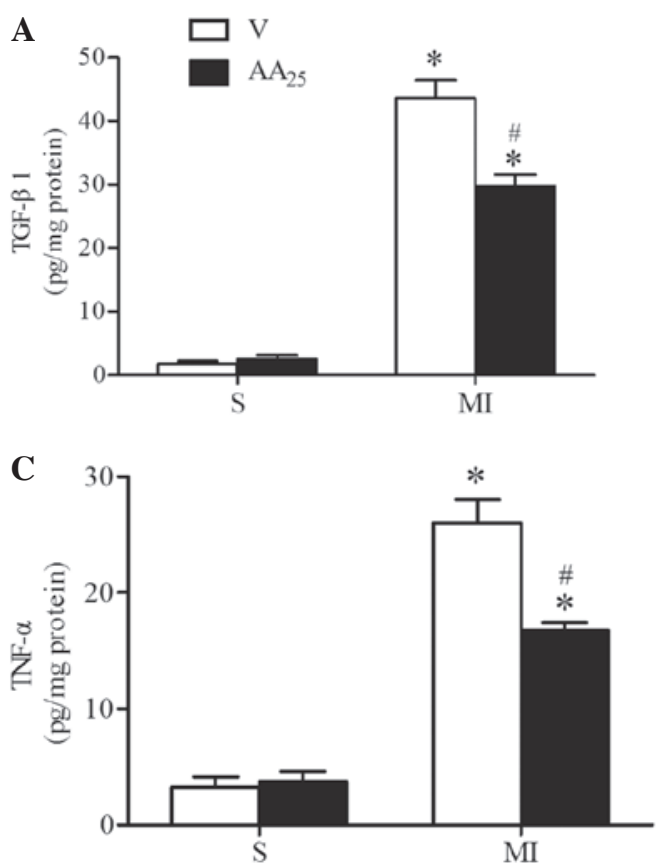
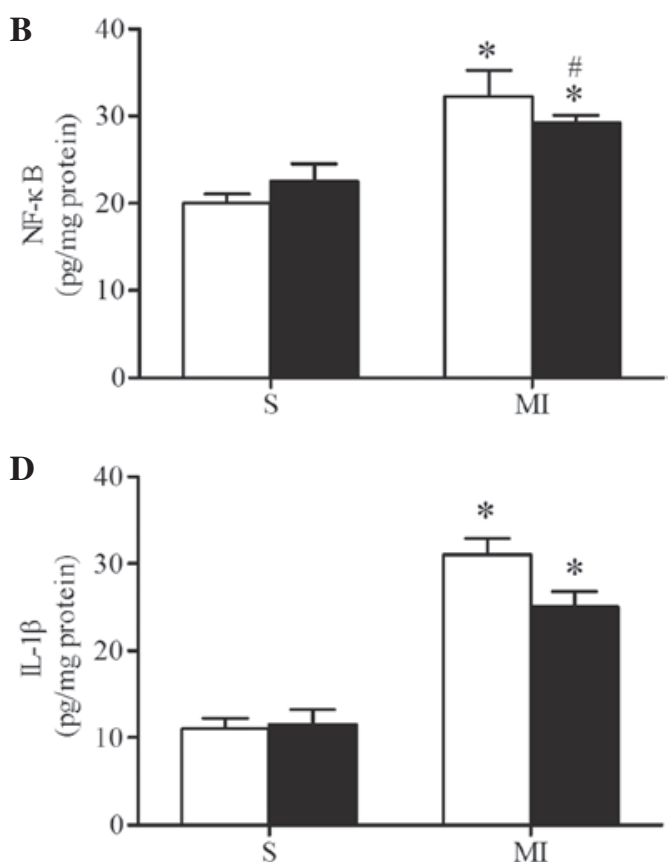

Figure 4. Expression levels of inflammatory cytokines in the infarct border zone were suppressed by AA after MI. Quantitative analysis of the protein expression levels of the inflammatory cytokines (A) TGF- $\beta 1,(B) \mathrm{NF}-\kappa \mathrm{B},(\mathrm{C}) \mathrm{TNF}-\alpha$ and (D) IL-1 $\beta$ in four groups (n=6-8 per group) at 4 weeks after MI, as determined using ELISA. Values are presented as the mean \pm standard deviation. ${ }^{*} \mathrm{P}<0.05 \mathrm{MI}$ vs. $\mathrm{S}$ group; ${ }^{*} \mathrm{P}<0.05 \mathrm{AA} 25$ vs. V group. V, vehicle; $\mathrm{S}$, sham; TGF- $\beta 1$, transforming growth factor- $\beta 1$; AA, asiatic acid, MI, myocardial infarction; NF- $\kappa \mathrm{B}$, nuclear factor- $\kappa \mathrm{B}$; TNF- $\alpha$, tumor necrosis factor- $\alpha$; IL- $1 \beta$, interleukin-1 $\beta$. ELISA, enzyme-linked immunosorbent assay.

\section{Results}

AA $(25 \mathrm{mg} / \mathrm{kg})$ exerted a protective effect on cardiac function after MI. To determine whether AA treatment was able to improve cardiac function following MI, and to clarify the appropriate dosage of AA for the further experiments, echocardiographic analyses were conducted at day 28 post-modeling (Fig. 2). Compared with the $\mathrm{S}+\mathrm{V}$ group, $\mathrm{MI}$ injury caused a significant reduction in LVEF $(\mathrm{S}+\mathrm{V}$ vs. $\mathrm{MI}+\mathrm{V}, 68.25 \pm 2.72$ vs. $31.50 \pm 1.04 \%, \mathrm{P}<0.05)$ and $\mathrm{FS}(\mathrm{S}+\mathrm{V}$ vs. MI $+\mathrm{V}, 40.25 \pm 1.32$ vs. $31.52 \pm 1.06 \%, \mathrm{P}<0.05)$. Treatment with $5 \mathrm{mg} / \mathrm{kg}$ AA produced no significant changes in cardiac 
A

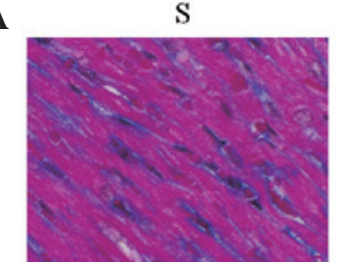

MI

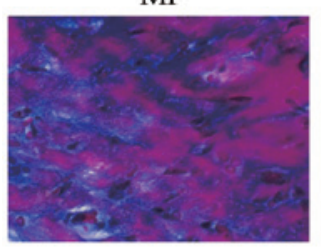

C

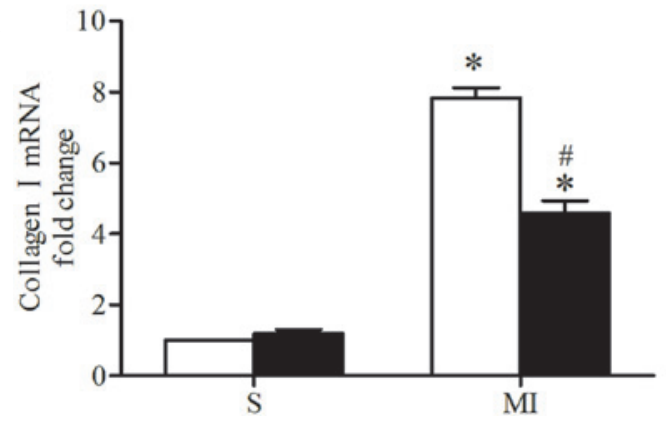

B

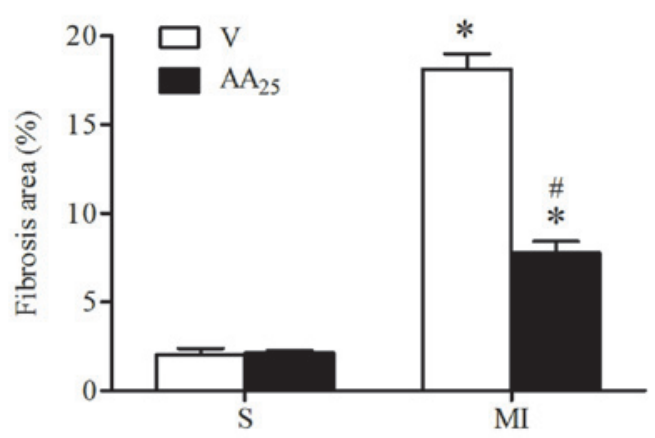

D

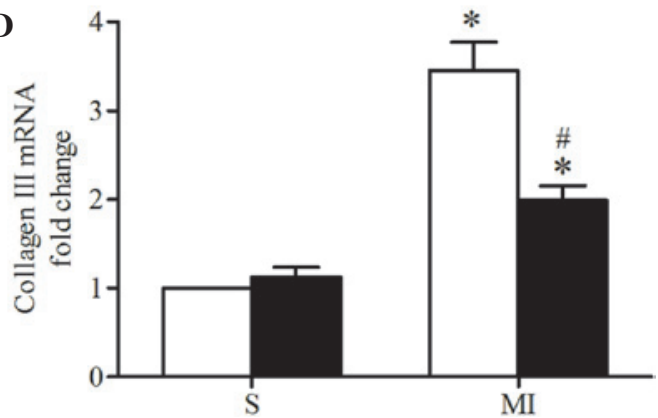

Figure 5. Post-ischemic myocardial interstitial fibrosis was prevented by AA administration. (A) Representative images of Masson's trichrome-stained heart sections from four groups ( $\mathrm{n}=6-8$ per group) at 4 weeks after MI modeling (magnification, x200). (B) Quantitative analysis of myocardial interstitial fibrosis. mRNA fold change of the expression of (C) collagen I and (D) collagen III. Values are presented as the mean \pm standard deviation. *P<0.05 MI vs. S group; ${ }^{\#} \mathrm{P}<0.05 \mathrm{AA}_{25}$ vs. V group. S, sham; AA, asiatic acid; MI, myocardial infarction; V, vehicle.

function compared with $\mathrm{MI}+\mathrm{V}$ rats. However, the $\mathrm{MI}+\mathrm{AA}_{25}$ and $\mathrm{MI}+\mathrm{AA}_{50}$ groups exhibited significantly improved cardiac function compared with the $\mathrm{MI}+\mathrm{V}$ group, as indicated by increased LVEF and FS. Additionally, $50 \mathrm{mg} / \mathrm{kg}$ AA did not have a greater cardioprotective effect than $25 \mathrm{mg} / \mathrm{kg}$ $\mathrm{AA}$ in the $\mathrm{MI}$ model rats $\left(\mathrm{LVEF}, \mathrm{MI}+\mathrm{AA}_{50} \mathrm{vs} . \mathrm{MI}+\mathrm{AA}_{25}\right.$, $44.00 \pm 2.27$ vs. $42.50 \pm 1.44 \%, \mathrm{P}=0.60 ; \mathrm{FS}, \mathrm{MI}+\mathrm{AA}_{50}$ vs $\mathrm{MI}+\mathrm{AA}_{25}, 31.75 \pm 1.11$ vs. $\left.29.53 \pm 0.85 \%, \mathrm{P}=0.20\right)$. AA exerted a protective effect on cardiac function following $\mathrm{MI}$ in a dose-dependent manner, and $25 \mathrm{mg} / \mathrm{kg}$ AA was selected as the dosage for the subsequent experiments. None of the three dosages of AA produced an effect on the echocardiographic parameters in the rats subjected to sham modeling.

AA prevented cardiac hypertrophy in response to $M I$. The $\mathrm{HW} /$ body weight ratio of rats subjected to MI was significantly increased compared with that of the sham rats (MI $+\mathrm{V}$ vs. $\mathrm{S}+\mathrm{V}, 4.16 \pm 0.21$ vs. $3.03 \pm 0.27 \% ; \mathrm{P}<0.05)$, and AA treatment partly reversed this change $\left(\mathrm{MI}+\mathrm{AA}_{25} \mathrm{vs} . \mathrm{MI}+\mathrm{V}, 3.54 \pm 0.16 \mathrm{vs}\right.$. $4.16 \pm 0.21 \%, \mathrm{P}<0.05$; Fig. 3 ). The lung wet weight/body weight ratio of the MI rats was increased compared with that of the sham rats $(\mathrm{MI}+\mathrm{V}$ vs. $\mathrm{S}+\mathrm{V}, 2.90 \pm 0.22$ vs. $2.25 \pm 0.13 \%$, $\mathrm{P}<0.05)$, and this change was suppressed by AA treatment $\left(\mathrm{MI}+\mathrm{AA}_{25}\right.$ vs. $\mathrm{MI}+\mathrm{V}, 2.49 \pm 0.09$ vs. $\left.2.90 \pm 0.22 \%, \mathrm{P}<0.05\right)$. AA $(25 \mathrm{mg} / \mathrm{kg})$ did not affect cardiac weight in sham rats. These data indicated that AA prevented cardiac hypertrophy and diminished pulmonary congestion induced by MI. H\&E staining and analysis of the cardiomyocyte cross-sectional area in myocardial sections of remote areas to the infarct revealed that MI led to marked cardiomyocyte hypertrophy $(\mathrm{MI}+\mathrm{V}$ vs. $\mathrm{S}+\mathrm{V}, 379.3 \pm 12.6$ vs. $\left.267.0 \pm 25.6 \mu \mathrm{m}^{2}, \mathrm{P}<0.05\right)$, and AA treatment suppressed the MI-induced cardiomyocyte hypertrophy (MI + $\mathrm{AA}_{25}$ vs. MI + V: $344.0 \pm 17.6$ vs. $379.3 \pm 12.6 \mu \mathrm{m}^{2}$, $\mathrm{P}<0.05)$.

AA suppressed the expression of inflammatory cytokines following MI. The levels of TGF- $\beta 1, \mathrm{NF}-\kappa \mathrm{B}, \mathrm{TNF}-\alpha$ and IL- $1 \beta$ were tested in left ventricular myocardial tissue lysates using ELISA kits (Fig. 4). The levels of NF- $\kappa$ B, TGF- $\beta 1$, TNF- $\alpha$ and IL-1 $\beta$ were markedly increased in the MI rats compared with the sham rats, indicating that MI resulted in the upregulation of proinflammatory cytokine levels. Treatment of the MI rats with AA markedly reduced the expression levels of TGF- $\beta 1$, $N F-\kappa B$ and $T N F-\alpha$, but had no significant effect on IL-1 $\beta$ expression. AA produced no effect on the levels of these inflammatory cytokines in sham rats.

AA treatment prevented post-ischemic myocardial interstitial fibrosis. Cardiac tissue sections were stained with Masson's trichrome to determine infarct size of the heart and myocardial interstitial fibrosis in the infarct border zone (Fig. 5A). Infarct size was comparable between the vehicle- and AA-treated MI rats, indicating that the degree of pro-fibrotic stimulation was similar. Mild or no fibrosis was detected in the sham rats, and MI resulted in significantly increased interstitial fibrosis $(\mathrm{MI}+\mathrm{V}$ vs. $\mathrm{S}+\mathrm{V}, 18.13 \pm 1.74$ vs. $2.03 \pm 0.73 \%, \mathrm{P}<0.05$; Fig. $5 \mathrm{~B})$. AA treatment markedly decreased the MI-induced fibrosis in the border zone $\left(\mathrm{MI}+\mathrm{AA}_{25}\right.$ vs. $\mathrm{MI}+\mathrm{V}, 7.78 \pm 1.33$ vs. $18.13 \pm 1.74 \%$, $\mathrm{P}<0.05)$. Consistent with these observations, collagen I and III mRNA expression levels in the border zone were significantly 

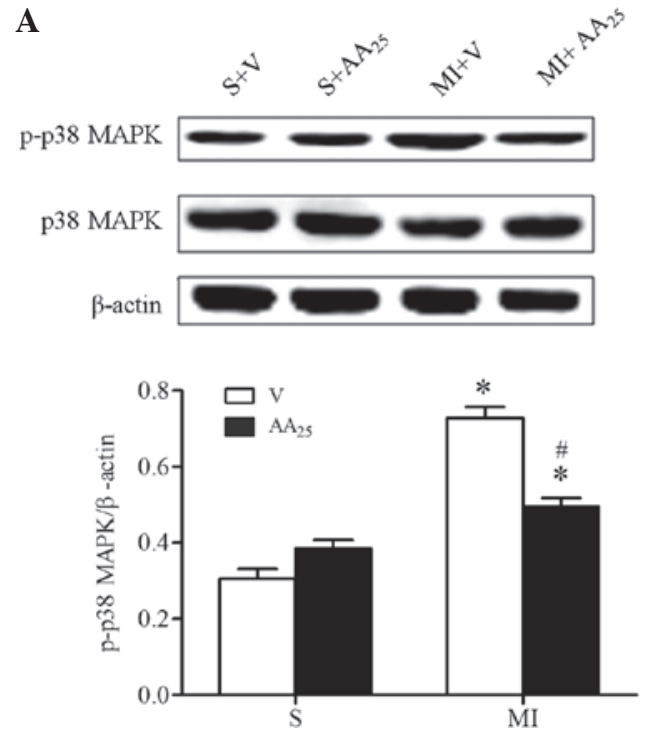

B
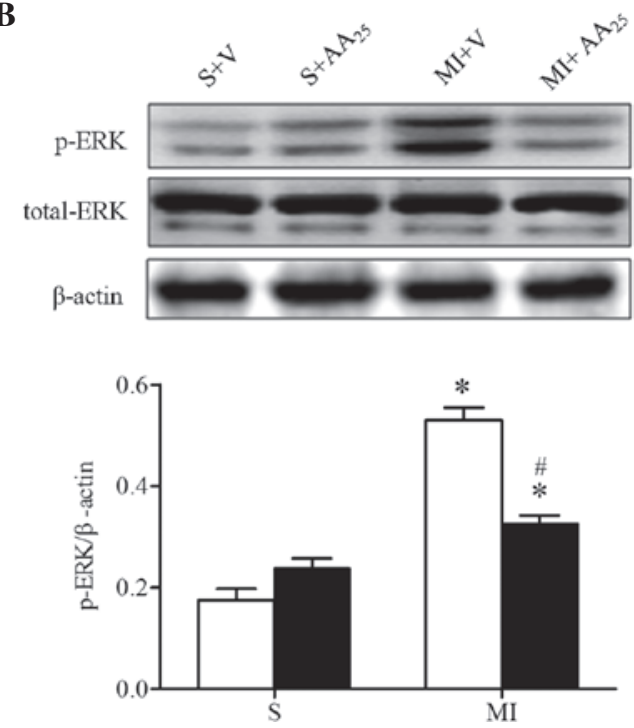

Figure 6. AA enhanced the phosphorylation of $\mathrm{p} 38 \mathrm{MAPK}$ and ERK1/2 in the infarct border zone after MI. Representative western blot analysis (upper panel) and densitometric measurements (lower panel) of (A) phospho-p38 MAPK and p38 MAPK, and (B) phospho-ERK1/2 and ERK1/2 expression levels in infarct border zone of the heart in four groups ( $\mathrm{n}=6-8$ per group). Values are presented as the mean \pm standard deviation. ${ }^{*} \mathrm{P}<0.05 \mathrm{MI}$ vs. $\mathrm{S}$ group; ${ }^{\prime \prime} \mathrm{P}<0.05 \mathrm{AA} 25$ vs. $\mathrm{V}$ group. p38 MAPK, p38 mitogen-activated protein kinase; S, sham; AA, asiatic acid; MI, myocardial infarction; V, vehicle; ERK, extracellular signal-regulated kinase.

increased in MI rats compared with sham rats, and the changes in the MI rats were reversed by AA treatment (Fig. 5C and D). AA did not affect cardiac collagen deposits in the sham rats. Collectively, these results demonstrated that AA prevented interstitial fibrosis in the myocardial infarct border zone following $\mathrm{MI}$ in rats.

AA inhibited p38 MAPK and ERK1/2 phosphorylation following $M I$. The aforementioned results suggest that AA suppressed the expression of inflammatory cytokines in addition to preventing interstitial fibrosis and myocardial hypertrophy following MI. To further investigate the molecular mechanisms underlying the effect of AA on the ischemic heart, the activation of p38 MAPK and ERK1/2 was determined, as they are reported to participate in cardiac remodeling (28). As expected, increased phosphorylation of p38 MAPK and ERK1/2 was observed in the border zone of hearts of MI rats compared with sham rats, and this change was reversed by AA treatment (Fig. 6). However, the total expression levels of p38 MAPK and ERK1/2 were not altered. AA did not affect the activation of $\mathrm{p} 38$ MAPK and ERK $1 / 2$ in sham rats. Collectively, the suppression of the p38 MAPK cascade and the inhibition of ERK1/2 phosphorylation may account for the beneficial effects of AA on hearts affected by MI.

\section{Discussion}

To the best of our knowledge, the present study is the first to show that AA treatment preserves cardiac function and alleviates left ventricular remodeling (30) in rats following MI (31), as indicated by the inhibition of cardiac hypertrophy, suppression of inflammatory cytokine expression and prevention of interstitial fibrosis in the infarct border zone. The inhibition of p38 MAPK and ERK1/2 phosphorylation may be an underlying mechanism of these effects. The present results indicate that AA may exert a therapeutic effect against cardiac dysfunction following MI.

Left ventricular remodeling is a complex process that occurs following MI, involving cardiomyocyte hypertrophy, inflammation and fibrosis (4-7). Initially following MI, fibrosis is necessary to rebuild the necrotic myocardium to preserve the structural integrity of the heart and to avoid cardiac rupture. However, the continuous remodeling of the infarct left ventricle may result in progressive ventricular dilation, decreased cardiac function and ultimately heart failure $(32,33)$. The extent of the detrimental remodeling is a predictive factor for morbidity and mortality following MI (34). At this stage, the collagen deposition in the infarct border zone is crucially involved and contributes substantially to the reduced myocardial elasticity and impaired cardiac contractility. Furthermore, hypertrophy and inflammation have been shown to promote myocardial fibrosis and left ventricular remodeling. Thus, strategies that inhibit cardiac remodeling following MI represent a major therapeutic goal of modern cardiology (35).

Recently, herbal compounds such as scutellarin and Schisandrin B have been demonstrated to produce beneficial effects against remodeling in the infarcted heart $(28,30)$. The results of the present study suggest that AA, a compound extracted from the herb Centella asiatica, may improve impaired cardiac function following MI. Previous studies have demonstrated that AA protects cultured cardiomyocytes against high glucose-induced injury (22) and exerts anti-hypertrophic effects in a mouse model of cardiac hypertrophy induced by pressure overload in vivo (21); the mechanisms underlying these effects may involve the ability of AA to attenuate oxidative stress and to suppress the protein expression of TGF- $\beta 1$, $\mathrm{NF}-\kappa \mathrm{B}, \mathrm{p}-\mathrm{ERK} 1 / 2$ and phospho-p38 MAPK. Although these studies clarified the effect of AA on these signal molecules closely associated with cardiac remodeling, it remained unclear whether the administration of AA was able to inhibit 
cardiac fibrosis and improve cardiac function following MI. The present study provides the first direct evidence showing that treatment with AA for 4 weeks significantly inhibits the deterioration of cardiac function and myocardial remodeling in a rat model of MI.

The molecular mechanism of left ventricular remodeling is complex, involving a number of biochemical intracellular signaling processes. TGF- $\beta 1$ is an inflammatory cytokine and a key profibrotic cytokine that is elevated in experimental MI, and anti-TGF gene therapy attenuates cardiac remodeling via reducing cardiac fibrosis (31). The present result indicating that AA inhibited the expression of TGF- $\beta 1$ suggests that the suppression of the TGF- $\beta 1$ pathway underlies the anti-remodeling activity of AA in post-infarct rat hearts. The p38 MAPK and ERK1/2 pathways, two classical cascades associated with cardiomyocyte hypertrophy, have also been demonstrated to serve a crucial function in the pathogenesis of cardiac remodeling (12-14). Ren et al demonstrated that daily treatment of rats with the p38 MAPK inhibitor RWJ 67657 resulted in alleviation of cardiac fibrosis and hypertrophy, in addition to improved cardiac performance following MI (36). In addition, Thum et al reported that the activation of ERK1/2 enhanced cardiac fibroblast proliferation and thereby promoted interstitial fibrosis and cardiac dysfunction (14). The present results revealed that the activation of p38 MAPK and ERK1/2 induced by MI was significantly inhibited by the administration of AA, as indicated by the reduced expression levels of phospho-p38 MAPK and phospho-ERK1/2. Therefore, the AA-mediated activation of p38 MAPK and ERK1/2 represents a potential mechanism underlying the beneficial effects of AA on impaired cardiac function following MI.

In conclusion, the results of the present study demonstrate that AA exerts notable benefits on impaired cardiac function and heart remodeling following MI, and that the suppression of TGF- $\beta 1$, phospho-p38 MAPK and phospho-ERK1/2 expression may underlie these effects. These findings suggest that AA therapy may be effective in the treatment of ischemia-associated heart diseases.

\section{Acknowledgements}

This study was supported by the National Natural Science Foundation of China (grant no. 2012BAJ18B01). The authors thank the Academy of Military Medical Science for their assistance.

\section{References}

1. Roger VL, Go AS, Lloyd-Jones DM, Benjamin EJ, Berry JD, Borden WB, Bravata DM, Dai S, Ford ES, Fox CS, et al: Heart disease and stroke statistics - 2012 update: A report from the American Heart Association. Circulation 125: e2-e220, 2012.

2. Opie LH, Commerford PJ, Gersh BJ and Pfeffer MA: Controversies in ventricular remodelling. Lancet 367: 356-367, 2006.

3. Biomarkers, genomics, telemetry, computational biology and zebrafish: Will one of these solve the problems of post-myocardial infarction heart failure? Eur Heart J 35: 57-58, 2014.

4. Khan R and Sheppard R: Fibrosis in heart disease: Understanding the role of transforming growth factor-beta in cardiomyopathy, valvular disease and arrhythmia. Immunology 118: 10-24, 2006

5. Mann DL: Mechanisms and models in heart failure: A combinatorial approach. Circulation 100: 999-1008, 1999.
6. Fosshaug LE, Berge RK, Beitnes JO, Berge K, Vik H, Aukrust P, Gullestad L, Vinge LE and Øie E. Krill oil attenuates left ventricular dilatation after myocardial infarction in rats. Lipids Health Dis 10: 245, 2011.

7. Yang F, Yang XP, Liu YH, Xu J, Cingolani O, Rhaleb NE and Carretero OA: Ac-SDKP reverses inflammation and fibrosis in rats with heart failure after myocardial infarction. Hypertension 43: 229-236, 2004.

8. Jugdutt BI: Aging and remodeling during healing of the wounded heart: Current therapies and novel drug targets. Curr Drug Targets 9: 325-344, 2008.

9. Nicoletti A, Heudes D, Mandet C, Hinglais N, Bariety J and Michel JB: Inflammatory cells and myocardial fibrosis: Spatial and temporal distribution in renovascular hypertensive rats. Cardiovasc Res 32: 1096-1107, 1996.

10. Bujak $\mathrm{M}$ and Frangogiannis NG: The role of TGF-beta signaling in myocardial infarction and cardiac remodeling. Cardiovasc Res 74: 184-195, 2007.

11. Zhang H, Wu J, Dong H, Khan SA, Chu ML and Tsuda T: Fibulin-2 deficiency attenuates angiotensin II-induced cardiac hypertrophy by reducing transforming growth factor- $\beta$ signalling. Clin Sci (Lond) 126: 275-288, 2014.

12. Muslin AJ: MAPK signalling in cardiovascular health and disease: Molecular mechanisms and therapeutic targets. Clin Sci (Lond) 115: 203-218, 2008.

13. Yeh CC, Li H, Malhotra D, Turcato S, Nicholas S, Tu R, Zhu BQ, Cha J, Swigart PM, Myagmar BE, et al: Distinctive ERK and p38 signaling in remote and infarcted myocardium during post-MI remodeling in the mouse. J Cell Biochem 109: 1185-1191, 2010.

14. Thum T, Gross C, Fiedler J, Fischer T, Kissler S, Bussen M, Galuppo P, Just S, Rottbauer W, Frantz S, et al: MicroRNA-21 contributes to myocardial disease by stimulating MAP kinase signalling in fibroblasts. Nature 456: 980-984, 2008.

15. Krishnamurthy RG, Senut MC, Zemke D, Min J, Frenkel MB, Greenberg EJ, Yu SW, Ahn N, Goudreau J, Kassab M, et al: Asiatic acid, a pentacyclic triterpene from Centella asiatica, is neuroprotective in a mouse model of focal cerebral ischemia. J Neurosci Res 87: 2541-2550, 2009.

16. Huang SS, Chiu CS, Chen HJ, Hou WC, Sheu MJ, Lin YC, Shie PH and Huang GJ: Antinociceptive activities and the mechanisms of anti-inflammation of asiatic acid in mice. Evid Based Complement Alternat Med 2011: 895857, 2011.

17. Ramachandran V, Saravanan R and Senthilraja P: Antidiabetic and antihyperlipidemic activity of asiatic acid in diabetic rats, role of HMG CoA: In vivo and in silico approaches. Phytomedicine 21: 225-232, 2014.

18. Bian D, Zhang J, Wu X, Dou Y, Yang Y, Tan Q, Xia Y, Gong Z and Dai Y. Asiatic acid isolated from Centella asiatica inhibits TGF- $\beta 1$-induced collagen expression in human keloid fibroblasts via PPAR- $\gamma$ activation. Int J Biol Sci 9: 1032-1042, 2013.

19. Dong MS, Jung SH, Kim HJ, Kim JR, Zhao LX, Lee ES, Lee EJ, Yi JB, Lee N, Cho YB, et al: Structure-related cytotoxicity and anti-hepatofibric effect of asiatic acid derivatives in rat hepatic stellate cell-line, HSC-T6. Arch Pharm Res 27: 512-517, 2004.

20. Tang LX, He RH, Yang G, Tan JJ, Zhou L, Meng XM, Huang XR and Lan HY. Asiatic acid inhibits liver fibrosis by blocking TGF-beta/Smad signaling in vivo and in vitro. PLoS One 7: e31350, 2012.

21. Si L, Xu J, Yi C, Xu X, Wang F, Gu W, Zhang Y and Wang X. Asiatic acid attenuates cardiac hypertrophy by blocking transforming growth factor- $\beta 1$-mediated hypertrophic signaling in vitro and in vivo. Int J Mol Med 34: 499-506, 2014.

22. Chan CY, Mong MC, Liu WH, Huang CY and Yin MC: Three pentacyclic triterpenes protect $\mathrm{H} 9 \mathrm{c} 2$ cardiomyoblast cells against high-glucose-induced injury. Free Radic Res 48: 402-411, 2014.

23. Orenstein TL, Parker TG, Butany JW, Goodman JM, Dawood F, Wen WH, Wee L, Martino T, McLaughlin PR and Liu PP: Favorable left ventricular remodeling following large myocardial infarction by exercise training. Effect on ventricular morphology and gene expression. J Clin Invest 96: 858-866, 1995.

24. Bunbupha S, Pakdeechote P, Kukongviriyapan U, Prachaney P and Kukongviriyapan V: Asiatic acid reduces blood pressure by enhancing nitric oxide bioavailability with modulation of eNOS and $\mathrm{p} 47 \mathrm{phox}$ expression in L-NAME-induced hypertensive rats. Phytother Res 28: 1506-1512, 2014.

25. Lee KY, Bae ON, Serfozo K, Hejabian S, Moussa A, Reeves M, Rumbeiha W, Fitzgerald SD, Stein G, Baek SH, et al: Asiatic acid attenuates infarct volume, mitochondrial dysfunction, and matrix metalloproteinase- 9 induction after focal cerebral ischemia. Stroke 43: 1632-1638, 2012. 
26. Araki S, Izumiya Y, Hanatani S, Rokutanda T, Usuku H, Akasaki Y, Takeo T, Nakagata N, Walsh $\mathrm{K}$ and Ogawa $\mathrm{H}$ : Akt1-mediated skeletal muscle growth attenuates cardiac dysfunction and remodeling after experimental myocardial infarction. Circ Heart Fail 5: 116-125, 2012.

27. Vahtola E, Louhelainen M, Merasto S, Martonen E, Penttinen S, Aahos I, Kytö V, Virtanen I and Mervaala E: Forkhead class O transcription factor 3a activation and Sirtuin1 overexpression in the hypertrophied myocardium of the diabetic Goto-Kakizaki rat. J Hypertens 26: 334-344, 2008.

28. Pan Z, Zhao W, Zhang X, Wang B, Wang J, Sun X, Liu X, Feng S, Yang B and Lu Y: Scutellarin alleviates interstitial fibrosis and cardiac dysfunction of infarct rats by inhibiting TGF $\beta 1$ expression and activation of p38-MAPK and ERK1/2. Br J Pharmacol 162: 688-700, 2011.

29. Zhu D, Wang H, Zhang J, Zhang X, Xin C, Zhang F, Lee Y, Zhang L, Lian K, Yan W, et al: Irisin improves endothelial function in type 2 diabetes through reducing oxidative/nitrative stresses. J Mol Cell Cardiol 87: 138-147, 2015.

30. Chen P, Pang S, Yang N, Meng H, Liu J, Zhou N, Zhang M, Xu Z, Gao W, Chen B, et al: Beneficial effects of schisandrin B on the cardiac function in mice model of myocardial infarction. PLoS One 8: e79418, 2013.
31. Okada H, Takemura G, Kosai K, Li Y, Takahashi T, Esaki M, Yuge K, Miyata S, Maruyama R, Mikami A, et al: Postinfarction gene therapy against transforming growth factor-beta signal modulates infarct tissue dynamics and attenuates left ventricular remodeling and heart failure. Circulation 111: 2430-2437, 2005.

32. Kurrelmeyer K, Kalra D, Bozkurt B, Wang F, Dibbs Z, Seta Y, Baumgarten G, Engle D, Sivasubramanian N and Mann DL: Cardiac remodeling as a consequence and cause of progressive heart failure. Clin Cardiol 21: I14-I19, 1998.

33. Hein S, Arnon E, Kostin S, Schönburg M, Elsässer A, Polyakova V, Bauer EP, Klövekorn WP and Schaper J: Progression from compensated hypertrophy to failure in the pressure-overloaded human heart: Structural deterioration and compensatory mechanisms. Circulation 107: 984-991, 2003.

34. White HD and Braunwald E. Applying the open artery theory: Use of predictive survival markers. Eur Heart J 19: 1132-1139, 1998.

35. Vanhoutte D, Schellings M, Pinto Y and Heymans S. Relevance of matrix metalloproteinases and their inhibitors after myocardial infarction: A temporal and spatial window. Cardiovasc Res 69: 604-613, 2006.

36. Ren J, Zhang S, Kovacs A, Wang Y and Muslin AJ: Role of p38alpha MAPK in cardiac apoptosis and remodeling after myocardial infarction. J Mol Cell Cardiol 38: 617-623, 2005. 